\title{
Kinematical features of locomotion -basis of fish control management in zones of influence of hydrotechnical structures
}

\author{
Pavel Mikheev 1,*, Vladimir Borovskoy ${ }^{1}$, Vladimir Vanzha ${ }^{2}$, and Kirill Belokur ${ }^{2}$ \\ ${ }^{1}$ Novocherkassk Engineering and Melioration Institute named after A.K. Kortunov of Don State \\ Agrarian University, Pushkinskaya street, 111, 346428, Novocherkassk, Russia \\ ${ }^{2}$ Kuban State Agrarian University named after I.T. Trubilin, 13 Kalinina str, 350044, Krasnodar, \\ Russia
}

\begin{abstract}
The structural analysis of the locomotion of various fish species presented in this work opens the possibility to take a fresh look at the solution of the problem of controlling fish behavior in a stream - through the prism of the kinematic structure of vortex structures generated by fish in the course of their swimming by different styles. In the locomotion of fish, there is an analogy with the phenomenon of the superimposition of two "antagonistic" forms of the jet stream - the wakeful (behind the streamlined object) and the flooded turbulent stream.
\end{abstract}

\section{Introduction}

Scientific support of ichthyofauna protection is based on accommodation of specific behavior of fish's particularities in the river stream. The study of the features of adaptation to movement in the aquatic environment for fish of various ecological groups is of interest in the theoretical and practical aspects of water management construction. However, the lack of knowledge of this kind restrains the solution of many acute environmental problems including the preservation and reproduction of fish stocks in conditions of regulation of river runoff and the increasing volume of water consumption from sources of fisheries management importance. The purpose of the study is to search for and develop methods for controlling the behavior of fish in the area of the hydraulic structure by changing the cum-mathematical structure of the water flow. For this, along with hydrodynamics, it is necessary to study the kinematic structure of the flow formed by the fish in the process of locomotion. Obtaining such information opens up the prospect of creating fish-protection zones (for example, near water intakes or in the lower reaches of hydroelectric complexes before entering the fish-passing structure), in which the movement of the fish will take place with minimal energy expenditure.

In addition to the biomechanical and hydrodynamic approaches to the study of fish locomotion, the kinematic approach is related to the inquiry of the geometric parameters of fish and the vortex structures formed by it. It is no coincidence that in the Western countries and in the USA, to the study of vortex formation in the process of swimming of fish and dolphins and its role in creating traction is given great attention [1]. This work is a continuation and development of the conclusions made by the authors earlier in this direction [2, 3]. It attempts to analyze the mechanism of swimming of fish from the

*Corresponding author: rekngma@magnet.ru 
viewpoint of the kinematic structure of the vortex structures formed by the fish.

Swimming of the fish is provided by the undulating movement of the body and the tail fin. At the heart of the driving (locomotor) functions, the formation of vortex structures and their interaction lie the unified principles of fluid mechanics [1-4], the knowledge of which makes it possible to divide fish into separate groups according to the type of navigation [5].

The principle of operation of any bioprocessor is based on one or another known form of organization of fluid motion, from which the basic styles of navigation flow: wave-like, oscillatory, reactive-jet, rowing.

The first principle consists in the difference between undulating and oscillatory motions.

In undulating motion, the locomotion wave passes along the propulsor - a structure (a part of the body with a group of muscles) that generates the driving force. Wavy movement is created by the body and the caudal fin acting as a single whole; fins connected to the body.

The movement of a biological object in water is closely related to the formation of a jet stream and vortex structures, the most typical of which is the fungoid, which precedes the initial stage of development of the jet [6]. From its characteristics depends largely on the speed of movement of fish.

\section{The principle of the wave propulsion}

The motion of a body of any shape in a viscous continuous medium is accompanied by the presence of a resistance, which consists of the frontal $(\mathrm{Cx})$ and surface resistance $(\mathrm{Cp})$. When flowing around a cylinder's flow, this manifests itself in the creation behind it of a rarefied rarefaction nucleus, behind which a plume of reduced velocities (a wake-up stream) is formed with a system of asymmetrically local (in checkmate order) local vortices-the Carman's prehistory. The opposite of this flow is a flooded turbulent jet, in which, unlike a wandering jet, a nucleus of increased pressure forms at the initial section.

Both forms of flow are basic. The dimensions of their initial sections (nuclei) are approximately the same both in width and in length [7, 8]. The superposition of these two "antagonistic" forms of jet flow (identical in size, but opposite in pressure) will lead to mutual-expression - hydrodynamic annihilation. Apparently, in practice, this principle is tried to realize the fish by performing coordinated wave and oscillatory motions.

Let us consider this in detail. The presence behind the streamlined object in the rarefaction nucleus, the pulsating circulating current, contributes to an increase in the total resistance. The "immobilization" of this region by connecting an uninterrupted streamline profile (the body of a fish) is the first step of nature in the way of reducing the overall resistance.

The next step is connected with the generation of transverse waves into the cycle of the body's own oscillations [8]. Harmonious oscillation of the wing of the bird (fouling) provides alternate formation of the boundary layer on each of the two sides, which helps to reduce the size of the streamlined area by about two times, and, consequently, the total resistance - the "waving wing" principle.

On the pressure (active) side of the wing, vortex structures are formed, which cause the surface resistance of the boundary layer. In water (as a denser medium compared to air), the formation of such vortices on the body of the fish creates more resistance than in birds in the air. Nevertheless, some species of vertebrates develop in the aquatic environment the same rate of linear displacement as terrestrial animals. Tuna, salmon, sharks develop a cruising speed within the range of $30-40 \mathrm{~km} / \mathrm{h}$. And such species as swordfish and blue marlin, swim at short intervals with a speed of over $100 \mathrm{~km} / \mathrm{h} \mathrm{[4].}$

Water inhabitants developed their own mechanism for reducing the vortex resistance. 
To do this, they make full use of the full potential of the wave. As a result of the bending and unbending of the trunk in the horizontal plane, a locomotion wave arises that performs several functions at once: it creates tractive force, localizes and transports vortices to the caudal fin (similar to dolphin skin damping), and ensures optimal caudal fin runout.

High-speed water animals use the energy of a push for water for linear movement. Fast fish are repelled by the trunk, tail stalk and tail fin. Such a mechanism of external kinematics is the basis for the movement of most active fish and represents the classical technique of swimming (herring, mackerel, marlin, shark) and has one of two names, depending on how much the body is involved. If a large part of the body is used for advancement (between one-half and two-thirds of its length), then the fish has a subcarangiform type of locomotion. If the muscles that move the body are moved to the last third of the body length (mostly the tail), then a carangiform type is obtained.

The next step is associated with a reduction in drag by modifying the shape of the head part of the fish. In general, the morphometric parameters that characterize the flowability of the body of a fish are due to a wide range of behavioral reactions and associated high-speed regimes.

The most important element of the mechanism by which to move forward is the shade of the fish in the profile. Fishes that use the movement of the body and the tail of the fin create a greater pull when the propulsion element has a greater height, and as a result, a maximum pull is created by fish with a high shade.

With continuous swimming, the effect of one element significantly affects the action of another element, on this interaction of the elements and determines which silhouette will ensure the greatest efficiency of motion.

When cruising (marathon) and sprint swimming, the movements that create traction are repeated cyclically. Because of these repetitive movements, continuous swimming can be considered as a periodic pushing. When cruising in a certain direction with a sufficiently high constant speed (tuna, mackerel, etc.) and sprinting with acceleration for short distances (many river fish), the amplitude of tail impacts is smaller than with intermittent navigation. The tail rarely travels over a distance exceeding $20 \%$ of the body length, which makes it possible to pass through the body a greater number of locomotion waves. Therefore, each rear element increases the acceleration reported to the water by the front element.

\section{Formation of lateral vortices}

The longitudinal force created by the propulsion elements of the body, which are closer to the tail, is greater than the force created by the elements located at the head. The reason for this is caudal deviation of the near-tail segments of the body in comparison with the okologalovymi segments. The second reason why the owl-tailed elements is more important for translational movement is that during the passage of the locomotion wave, the posterior part of the body, bending, moves to a greater distance than the anterior one. Therefore, the velocity of transverse movement of the near-tail elements is greater, and they impart a greater acceleration to the water than the elements of the front part of the fish body.

In fishes with an elongated body, the length of the locomotion wave $(\lambda)$ and its phase velocity $(V)$ remain constant all the time: the amplitude of body oscillations $\left(a_{0}\right)$ from head to tail does not increase $\left(V=\right.$ const; $\lambda=$ const; $a_{0}=$ const $)$. Angular type of movement provides low speeds of movement, but it gives advantages in power, because it is more economical.

The hydrodynamic approach allows us to conclude that a large traction force arises from the transfer of energy in space with the help of a low-speed wave (for example, rays are an 
example). In this case, a unidirectional energy transfer is created not by means of a moving mass (jet), but by a wave. Water in the cavity of the wave moves at a speed V from the head to the tail fin. For fish whose height and body thickness decrease from head to tail, respectively, the wavelength and amplitude will be variable ( $\lambda=\operatorname{var} ; a_{0}=$ var ), The locomotion wave from the head to the caudal fin increases. With a constant volume of water in the cavity of the wave, its shape varies from high and low to high and wide. Transformation of the volume is accompanied by the unwinding of water in it (with simultaneous movement to the opposite side) and ends on the stem of the caudal fin. Here, the height of the lateral (locomotor) vortex reaches its minimum, equal to the height of the caudal peduncle $\left(h_{\mathrm{BV}}=h_{\mathrm{CX}}\right)$.

Running along the body of the fish, the locomotion wave, dragging water (in the valleys), forms a jet stream with maximum velocities on the longitudinal axis. Since the velocity of propagation of the wave along the body $\mathrm{V}$ exceeds the velocity of displacement of the fish $\left(V>U_{\mathrm{p}}\right)$, untwisting of the vortex occurs in the same direction as the large eddies in the turbulent jet, that is, opposite to the tangent motion of the propulsor.

Since the body of the fish narrows toward the tail in the sagittal section, in the wave impingement (at the contact with the vortex structure), the body's height is on one side larger than the other, which causes a two-sided (above and below) fluid overflow and the formation of a symmetrical pair of longitudinal vortices. This is facilitated by the rarefaction that occurs behind the stem and caudal fin.

As a result, a vortex structure with mirror symmetry relative to the frontal plane is formed, consisting of two paired toroidal vortices with a common vertical axis of rotation. It receives liquid through two suction funnels (poles), located above and under the vortex on a common vertical axis.

During the analysis of the stages of formation of a vortex ring before decay, it is established that a similar vortex structure is formed when the toroidal vortex ring is lost as an intermediate (secondary) ring [9]. At the end of the wave, the formation of the lateral vortex ends at the maximum bend of the tail. At this stage, it represents two paired toroidal rings with a common axis of symmetry. Its kinematic structure is described in detail in [9].

The lateral vortex, untwisted by the undulating movement of the fish, is drawn into the zone of rarefaction behind the caudal fin. In the process of oscillatory motion, its outer layers are injected into a vortex sheet that descends from the pressure side through the trailing edge of the tail fin. In the result, two threads, dispersed in different ways (wave and cumulative effect), merge into one. At the time of the change in the direction of rotation of the outer layers of the lateral vortex after injection, the remaining part of the lateral vortex receives an additional impulse with the maximum spread of the tail fin and is discarded from it in the direction opposite to the movement of the fish.

At this moment, the force of pressure on the remaining part of the lateral vortex, which continues to rotate by inertia, increases sharply, which contributes to the creation of a traction force pulse. Thus, the undulation movement, apart from creating the thrust, contributes to the formation of lateral support vortices with a vertical axis of rotation, and the oscillatory motion ensures repulsion from them.

At the moment of extreme deviation of the caudal fin, the direction of rotation of the remaining part (core) of the lateral vortex coincides with the rotation of the vortex in the wake of the streamlined object - as in the path of Karman. After a change in the movement of the fin, the lateral vortex receives an additional pulse, which, through its outer layers, changes the direction of rotation of the liquid in the vortex body to the opposite, as in a turbulent jet. The interaction of two differently directed flows in one vortex - strengthens the ef- fect of recoil (according to the principle of "aikido"), the vortex slows down its movement. 


\section{Analysis of fins}

In the hydrodynamics of fish, the caudal fin is not regarded as a special specialized propulsor, all parts of the fish body contribute to the general movement, and the caudal fin according to the force properties is one of the parts of the general distributed motor system of the fish $[1,4]$.

For fish using only a locomotion wave (eels, moray eels), there is no need for a caudal fin (as a propulsor). Most of the carp-shaped ones have a flexible tail fin, which is why, under the action of a lateral force counteracting transverse movements, the caudal fin takes a concave shape during movement [10]. In addition to contributing to the creation of traction, the tail fin provides the fish with high maneuverability at high speed. So the crucian with the help of the caudal fin easily makes a turn by $90^{\circ}$ for one movement of the tail.

Two pectoral fins, as a rule, most actively used, are intended for coordinated movements. Sharks are needed as rudders, especially for moving up or down, while a significant part of other fish still use pectoral fins for jerking and forward movement (ostracisiform) 3 [11]. A classic example of the latter are representatives of the superfamily Ostraciidae. For example, a globefish is a typical taxonomic unit of this superfamily. Their key feature is the transfer of the function of the rudder to the caudal fin, while the pectoral fins push the body through the water column.

Blowers and bodies use dorsal and anal, rather than pectoral fins, to advance the body forward. This technique, known as balistiform, is rare. Nevertheless, it can be observed in the Lagoon triggerfish (Rhinecan-thus aculeatus), or in the sunfish (Mola). It moves slowly, mainly using the dorsal and anal fins, because in the process of evolutionary development, the caudal fin has been lost.

For a mixed propeller type, the morphometric parameters of the fish must satisfy the requirements of both forms of thrust creation. So the presence of a high tail stem, indicates the possibility of using the body of a fish as a wave propulsion, and the presence of a dredge in the tail fuselage-like a waving wing.

Representatives of the mackerel family have a caudal fin in the form of a Latin letter V, or, in the language of aerodynamics, a "swept wing". For high-speed fish, the pulling force is created mainly by the movements of the caudal fin, which is $\mathrm{V}$-shaped or approximated to the crescent. The half-angle between the elements of $\mathrm{V}$ is from $50^{\circ}$ to $60^{\circ}$ [10].

The last tail element defines a "clean" acceleration, which is associated with water, so the tip of the tail should be as wide as possible in the vertical direction. From the shape of the caudal fin, the structure of the current, formed in the wake of the fish, depends. The presence of a V-shaped notch accelerates the jet stream due to its compression (like the cumulative effect). Such, decorated (by V-shaped notch of the tail convoy) compressed jet stream, ideally approximating the velocity profile of the turbulent jet.

\section{Analysis of the vortex structures formed by the fish in the process of swimming}

The pressure surface of the caudal fin, completing close to harmonic oscillations at a variable angle of attack [4], completes the formation of a jet stream, which, coming off the tail fin, forms a two-dimensional mushroom-shaped vortex structure with frontal (horizontal) symmetry in the wake of the fish. Running down from the caudal fin, trapped in a stationary environment, exfoliates - formed a two-dimensional mushroom-prominent structure of two mirror-symmetrical vortex bundles, with horizontal axis of rotation. A rapid increase in the transverse dimensions of this structure leads to a sharp inhibition.

In total, the fish forms at its extreme points - lateral vortices with a vertical axis, and in 
the space between them - a symmetrical pair of transverse vortices with horizontal axes of rotation. In general, the rotation of two orthogonal pairs of vortices in space coincides in direction with the rotation of large vortices in an axisymmetric turbulent jet. The unidirectionality of coherent structures and the mutual proximity of single-sided (suction) poles at the ends of vertical and horizontal vortices at the point of change of the direction of motion of the tail fin indicate the possibility of their unification into a single toroidal vortex (ring).

The difference between the jet stream, formed by the movements of the confuser part of the fish, from the turbulent jet formed at the initial section, is primarily due to the cyclic generation of lateral vortices. As a result, in the wake of the fish, the vortices are staggered - as in the path of Karman, with the only difference being that they rotate in the opposite direction, that is, as in a turbulent stream. Rotation of the vortex core continues for some time by inertia in the direction specified initially by the locomotor wave. The outer (coaxial) layers of the vortex are curved by the caudal fin later in the opposite direction. The presence of two oppositely directed coaxial and axial circulation currents in the body of one vortex is accompanied by rapid damping, and if their potentials are equal, the whole vortex structure is immobilized. This can be compared with the phenomenon of annihilation (known from nuclear, quantum physics).

The cyclic nature of fish movements explains the fact that the formation is not complete (as, for example, in a squid or jellyfish), but a truncated jet ("half-jet"). Outwardly such a flow looks like the path of Karman, but, in fact, is a turbulent jet with rapidly damped vortices [10].

In the case when the diameter and circulation of the vertical and horizontal vortices in the wake of the fish coincide in magnitude $d_{\mathrm{BV}} \cong d_{\mathrm{GV}}=$ const, The projection in the axial plane of the joint axes of all vortices will tend to a circle. In this case, the distribution of the load on the body of the fish and the caudal fin will be optimal - a balanced mushroomshaped vortex structure is formed. It is characteristic and can serve as a kind of standard when comparing the styles of fish's swimming.

So for the eel (moray eels) the supporting vortices are the lateral vortices with the vertical axis of rotation; Horizontal vortices are absent. For high-speed fishes with a narrow stem of the caudal fin, horizontal vortices are vice versa, and vertical vortices are poorly developed (due to the thin stalk of the caudal fin). Between these extreme forms on the general scale are the remaining styles of swimming fish.

Support vortexes, having descended from a fish, are transformed into a single mushroom-like structure and are sharply braked like a parachute, straightened in the air. Ideally, the fish is removed from the hindered supporting mushroom structure at a rate equal to the velocity of the large vortex moving in the turbulent jet at the point of stability loss, that is $U_{p} \approx u_{p u}=u_{\text {orc }}, u_{p u}$ - velocity of the jet at the point of loss of stability; $u_{\text {orc }}$ - speed of movement of the supporting mushroom structure.

The supporting mushroom structure is created by the frontal (frontal) part of the jet stream, whose velocity approximately coincides in magnitude with the phase velocity of the locomotive wave $\mathrm{V}$, which in the turbulent jet corresponds to the maximum velocity in the cross section of the dynamic axis, it means $V \approx u_{m}$.

On the other hand, according to the results of the studies published in [9], the relative velocity of the jet at the point of loss of stability $\left(\sigma_{\mathrm{pu}}\right)$ is almost constant

$$
\sigma_{\mathrm{pu}}=u_{\mathrm{pu}} / u_{m} \approx 1 / F=0,618, F=\frac{1+\sqrt{5}}{2}=1,618 \ldots-\text { number phi }
$$


In the event that the inhibition of the characteristic supporting mushroom structure in the wake of the fish occurs instantaneously, the following proportion takes place:

$$
\frac{U_{p}}{V}=\frac{u_{o r c}}{V} \approx \frac{u_{p u}}{u_{m}} \approx \frac{1}{F}=0,618 .
$$

This value is confirmed to a certain extent by Gray's research [11, 12]. Measuring the speed of locomotion $U_{\mathrm{p}}$ This value is confirmed to a certain extent by Gray's research [11, 12]. Measuring the speed of locomotion (relative to the bottom of the aquarium) and the phase velocity of the motor wave $\mathrm{V}$ (relative to the body) for single stationary flows of different fish, Gray drew attention to the property of small variability in the ratio of these velocities, interpreting the ratio $U_{\mathrm{p}} / V$ as the slip of the locomotion wave with respect to the body water. Despite the great differences between the two velocities $U_{\mathrm{p}}$ and $\mathrm{V}$ the magnitude of their ratio turned out to be approximately constant: $U_{\mathrm{p}} / V=0,6 \pm 0,1$ and close to the value obtained on the basis of the analysis of the occurrence of large eddies in a turbulent jet [9].

The speed of swimming, the maneuverability and the very life of the fish indirectly depends on the stability of the support from which it repels, so the formation of a supporting mushroom structure that will be quickly decelerated (after dropping from the tail) is an evolutionary necessity. Therefore, the manner of the formation of traction largely determines the shape of the body most appropriate to the needs of this type of fish.

Some families of fish, having modified the scomboid type of motion, can develop the greatest observed speed in fish. Typical representatives acquired in the process of evolution sickle-tail and powerful muscles, which can drive it. They almost once divided the structure to create a jerk when moving and creating traction. This division was achieved due to a change in the shape of the body - the contraction of the tail part to the caudal fin [10].

The optimal shape of the body for periodic swimming involves thinning the body in front of the tail with the formation of a narrow tail (stalk), to which the tail is attached. This feature is characteristic of fish that spend most of their life in cruising. Above this speed, the energy balance is disrupted - the plume of the stream behind the fish lengthens, energy is quickly consumed.

The most common seafarers are, as a rule, owners of the tunnel type of locomotion (thunniforms). A classic example is tuna, in honor of which this type of movement is named. The shape of the tuna body is most closely approximated to the optimum for continuous swimming. The narrow tail part makes possible a less mobility of the anterior part of the body and contributes to the creation of a viable, ten-shaped body that reduces water resistance [5]. Tuna is a champion among high-speed swimmers for long distances. Using barely noticeable movements of the body, he controls a large crescent-shaped tail. The motor of the tail is due to the developed musculature. But it's not just the tail. The tail strokes determine up to $85 \%$ of the movement of the anterior part of the body with subcarangiform, carangiform and tunniform types of locomotion.

However, other fins also perform a number of important functions. In particular, the dorsal fin serves to make sharp turns and braking. Fish, which have a second dorsal fin, use it also to make a leap forward. In some cases, it is $15 \%$ enforceable. Anal fins act as a brake, the abdominals provide both inhibition and ascent, while the thoracic fins help to make quick turns. The lateral force at the end of the tail tends to cause lateral deflection of the front part of the fish body or its oscillatory movements. During periodic swimming due to lateral force consumes a large amount of energy. As the body height decreases, the side force decreases before the tail. The increase in the mass of the head part of the body 
according to Newton's second law, too, entails suppression of lateral vibrational movements. The resistance of the anterior part of the body to the action of the lateral force also increases with the help of a fin located on the upper surface of the body, which explains the presence of the anterior medial fins [5].

\section{Functional-morphological model of fish swimming types}

According to [5], in fish there are three basic mutually exclusive forms of the body, optimal for different types of swimming. In most fish, the locomotor apparatus consists of combinations of elements characteristic of specialized forms. Therefore, from the point of view of locomotor adaptations, most fish belong to "generalists", rather than "specialists". Despite the fact that only a few fish have a body shape that is close to optimal for a certain type of swimming, the principle of the three basic forms is observed. All morphofunctional variants of the locomotor apatate of fish are enclosed in a figure having the form of a triangle, the vertices of which correspond to the three basic forms of the body [5]. However, despite the conventionality of this scheme, most fish find their place here, determined by a combination of elements of specialized forms inherent in this species (Figure 1).

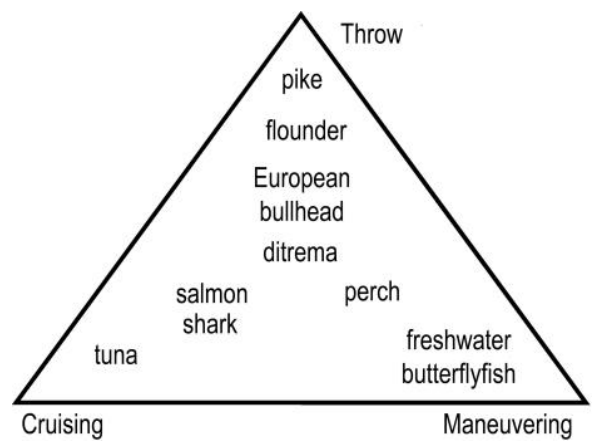

Fig. 1. Functional-morphological planar model of types swimming of fish [5].

The segments of the triangle correspond to groups of fish with different lo-comotor features, which constitute a certain continuum based on the difference in the nature of the resources used. One of them is the success of the attack on the victim. Tuna occupying the left corner of the triangle. The shape of the tuna body, which is very effective for cruising, limits the fish's ability to throw and maneuver. However, with a long swim at high speed, the tuna can meet more potential casualties and commit more attacks.

Fish, similar in shape to the body with a pike, located in the upper corner of the triangle. Since the pike is not capable of prolonged cruising, it is forced to wait for prey, and the number of encounters with victims is limited. Fish such as trout and perch occupy an intermediate position on the success of attacks and, being "generalists", are also capable of long swimming in search of prey. The combination in the form of a body of various elements gives these fish the possibilities for nutrition, close to those that are available to "specialists".

The creation of the driving force and the overcoming of the hydrodynamic resistance makes the fish possessing an elongated body shape more suitable for casting swimming, but possessing a different form of body - for cruising or maneuvering navigation [13]. These are the three main types of swimming. In some fish, the body shape is adapted to one of them. From the hydrodynamic and kinematic point of view, in addition to the three main body shapes shown in Figure 1, two more functionally opposite forms of fish - eel and swordfish - are characteristic. 
The movements of the eels show an anguilliform type of locomotion (an-guilliform). The pectoral fins can still be preserved and play an insignificant role in the movement. The tail of the eels can be tenacious. For example, a moray eagle, is capable of being thrown out of its lair with lightning speed and retreating as quickly as possible, taking prey with it. In this case, the body is used for coupling with a stationary object in the burrow, which increases the traction force. Holders of the anguilliform type locomotion hydrodynamic ideally suited for both forward and backward movements.

A swordfish can develop a speed of over $100 \mathrm{~km} / \mathrm{h}$. Judging by all, she is best able to solve the main hydrodynamic problems: reduce drag; quickly transfer the displaced liquid from the pressure diffusive part of the body to the dilute confusor, form a jet with the caudal fin "slamming" the space behind it with rapidly retarding supporting mushroom structures. It is the rate of inhibition of the vortices around the fish and in the wake behind it is the main indicator of the efficiency of the bioreactor.

Entering and leaving the unperturbed water space with minimal disturbance of the medium, with minimal energy expenditure, is the main indicator of the evaluation of the efficiency of the movement of fish at the given speed. Apparently, periodic swimming with sprinter speed corresponds to the upper boundary at which energy expenditure, body shape and degree of disturbance of the aquatic environment are brought to the ultimate state.

The five forms of the body of the fish are correlated with the kinematic and dynamic features of the locomotion described above. In the "continuum" between them are all the other forms and styles of swimming fish. Figure 2 shows graphically the kinematicmorphological model of fish.

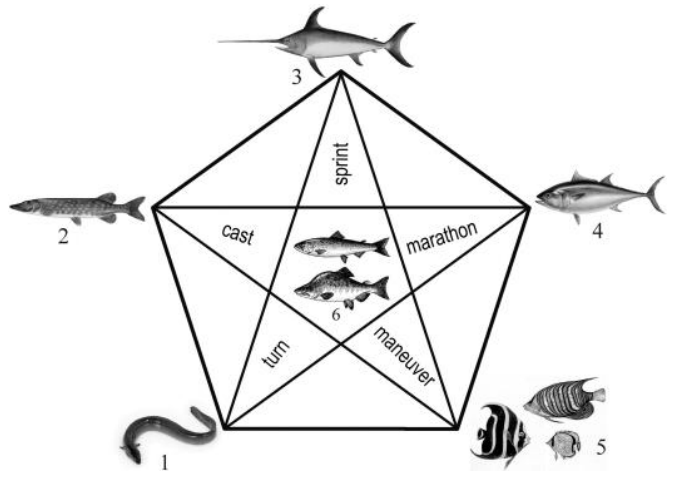

Fig. 2. Kinematic-morphological model of fish body shapes: 1 - eel; 2 - pike; 3 - swordfish; 4 - tuna; 5 - butterfly fish; 6 - salmon (pink salmon).

As a reference point in the central part, there are salmonids, characterized by a subkarangiiform type of locomotion. Features of their external structure allow a long time to swim straight through the lake or up the rivers. They can make quick maneuvers in case of attack by predators, during hunting for prey or fighting against constantly changing currents.

On the basis of the above structural analysis of the peculiarities of the fish's locomotion, the authors developed a kinematic-morphological model for the types of propulsion of biological objects in the aquatic environment (Figure 3). 


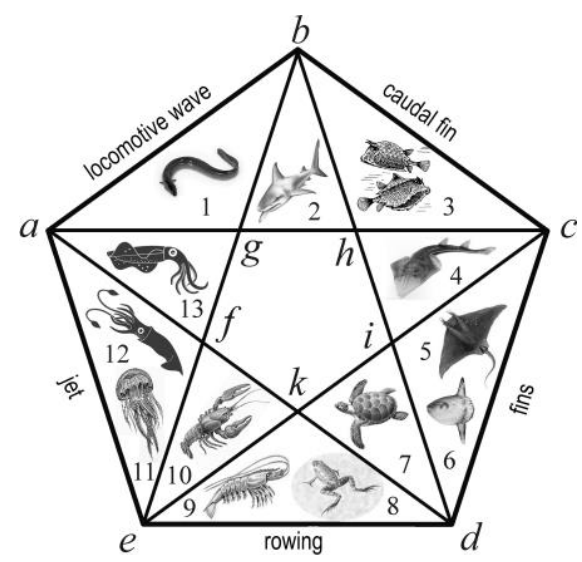

Fig. 3. Kinematic-morphological model of the types of propulsion of biological objects in the aquatic environment: 1 - eel; 2 - shark; 3 - bodies (ostraciidae); 4 - the Guitarfish (rhinobatus); 5 - manta ray (manta birostris); 6 - sunfish; 7 - sea turtle; 8 - frog; 9 - shrimp; 10 - cancer; 11 - jellyfish; 12 - squid; 13 - cuttlefish.

The "continuum" of the geometric model is limited to five basic types of bio-agents in the aquatic environment: "locomotion wave" - side ab; "oscillation" (the method of creating a jet with the oscillation of the tail fin is - bc, all other active - cd); "rowing" - de; the jet is ea. Each type of propulsor is correlated with biological representative ("specialist"), for: locomotion wave - it's eel, moray (abg); oscillation with caudal fin - body (bch); oscillations with other pairs and unpaired fins - stingray (manta), fish-moon, sea horse (cdi); rowing - shrimp, frog (dek); jet stream - jellyfish, squid (eaf).

Between the two adjacent "experts" in each of the ends of the five-coal star is a representative of the combined propulsion. So between the eel (locomotion wave) and the body (tail fin) is a shark - a representative of the classic (scomboid) type of swimming; between the body and the slope (the fish-moon) - the scrounge (the middle between the shark and the slope); Between the ramp and the shrimp (frog) - amphibians (sea-turtle, penguin); between a shrimp (frog) and squid (jellyfish) - cancer (with a rapid bend of the abdomen, a powerful push throws the cancer back, forming a mushroom structure that is the frontal part of the cummulative jet); between squid (jellyfish) and eel - cuttlefish, which has wings-fins that can act independently, giving it greater maneuverability. This model gives a clear idea of the interdependence at the kinematic (vortex) level of the basic types of propulsors used by biological objects in the aquatic environment.

Locomotion of fish in a motionless and mobile aquatic environment (river) has some differences. In an undisturbed environment, in the process of swimming, the fish expends energy to create vortex structures. Being in the turbulent environment of the river flow, it is forced to react to existing active vortex structures and use them rationally for their movement. It is no coincidence that in river fish the lateral receptors (lines) are more developed than in marine ones. This indicates their greater sensitivity to external pulsations of pressure [14], which can be described as "hydro-dynamic vision."

\section{Conclusion}

An analysis of the characteristic features of the dynamics and morphology of fish, causing a reduction in the total resistance, is performed. The basic types of fish locomotion are considered. The mechanism of formation of a lateral (lo-comotor) vortex and vortex structures formed by a fish in the process of navigation is described. A basic constant is established, which makes it possible to determine the translational velocity of the fish 
relative to the reference vortex structure (1). The functions of active and passive fins are described.

A kinematic-morphological model of fish body shapes has been developed (see Figure 2), with the help of which it becomes possible to establish a swimming style, which, in turn, determines the parameters of characteristic vortex structures that can be used as the basis for the formation of optimal to attract fish (for example, to the entrance to the fish-boat) or vice versa destructive - for scaring (from water intake). Based on the analysis of fish locomotion features, the authors developed a kinematic-morphological model of the types of propellers of biological objects in the aquatic environment (see Figure 3). Filling these two models is a perspective study.

\section{References}

1. E.V. Romanenko, S.G. Pushkov, Fundamental and applied hydrophysics 2, 13-28 (2008)

2. V.P. Borovskoy, Materials Vseros. scientific-practical. Conf. "Current state of the problem of fish protection and fish passage, their role in the conservation of aquatic biological resources" (Novocherkassk, 2010)

3. V.P. Borovskoy, P.A. Mikheev, Izvestiya VNIIG im. B.E. Vedeneeva 261, 89-98 (2011)

4. E.V. Romanenko, Hydrodynamics of fish and dolphins (KMK publishing house, Moscow, 2001)

5. V.N. Koleskin, V.V. Koleskin, Yaroslavl Pedagogical Bulletin. Natural. Science III-1, 48-60 (2011)

6. M. Van Dyke, Album of flows of liquid and gas: Trans. with English (Mir, Moscow, 1986)

7. G. Abramovich, The theory of turbulent jets (Nauka, Moscow, 1984)

8. G. Schlichting, L.G. Luoyqiang, The theory of the boundary layer (Nauka, Moscow, 1969)

9. P.A. Mikheev, V..P Borovskoy, The results of science. Issue 18. Selected Works of the All-Russian Conference on the Problems of Science and Technology (RAS, Moscow, 2015)

10. J. Lighthill, Mathematical Biogidodynamics (Institute of Computer Science, Izhevsk, 2014)

11. J. Gray, J. Exp. Biol. 12, 88-103 (1933)

12. J. Gray, J. Exp. Biol. 13-2, 192-199 (1936)

13. P.U. Webb, In the world of science 9, 34-48 (1984)

14. D.S. Pavlov, Biological fundamentals of controlling the behavior of fish in the flow of water (Nauka, Moscow, 1979) 